\title{
REFERENCE ODF CALCULATIONS FOR THE TORSION OF FCC RATE SENSITIVE POLYCRYSTALS
}

\author{
P. GILORMINI, L.S. TÓTH ${ }^{1}$ and J.J. JONAS ${ }^{2}$ \\ Laboratoire de mécanique et technologie, \\ Ecole normale supérieure de Cachan, CNRS, Université Paris VI, \\ 61 Av. Président Wilson, 94230 Cachan, France
}

\begin{abstract}
Analytic calculations are presented for the deformation induced ODF in a sheared FCC polycrystal. The uniform strain (Taylor) hypothesis and the theory of rate dependent slip are used. The fibre considered contains three interesting ideal orientations, and periodic $O D F$ variations are observed. The present predictions, which can be readily and rapidly obtained, are useful for testing computer codes for the simulation of large deformation textures.
\end{abstract}

\section{INTRODUCTION}

The development of deformation textures is usually simulated by using incremental procedures in which large numbers of individual grains and strain steps are treated by computer. The incremental nature of such simulations, however, leads to limitations in precision as a result of the appreciable number of steps required to attain large applied strains. Moreover, the orientation distribution function (ODF) is not easily deduced from these results for discrete orientations.

In the present study, the possibility is considered of predicting ODF's for the torsion of polycrystals by non-incremental analytical or semi-analytical means. Although limited to special situations, such results are of interest for the precise testing of computer-based incremental simulations. Moreover, the details of texture changes such as texture transitions and periodicity can be observed, which are not evident from the usual computations.

To the authors' knowledge, only one such analytical approach can be found in the literature, and it concerns a very special case. For a rate insensitive polycrystal to which the uniform strain (Taylor) model is applied. Wierzbanowski and Clément ${ }^{1}$ showed in 1984 that the ODF increases exponentially for (isolated) stable orientations when the divergence of the orientation velocity is negative. Similarly, positive divergences lead to .ODF intensity decreases. Here, rate sensitivity is taken into account, and a whole orientation fibre is analyzed.

\section{PRELIMINARY DEFINITIONS}

A polycrystal consisting of face centered cubic (FCC) crystals deforming by means of $\{111\}<110\rangle$ slip is considered and submitted to simple shear. The direction of macroscopic shearing is denoted by the index 1 , the normal to the shearing plane by 2 , and the direction perpendicular to 1 in this plane by 3, see Figure 1a. (In the case of the fixed end torsion of a

\footnotetext{
${ }^{1}$ Institute for General Physics, Eötvös University, 1445 Budapest, P.O.B. 323, Hungary.

2 Department of Metallurgical Engineering, McGill University, 3450 University Street, Montreal, H3A 2A7 Canada.
} 
cylindrical specimen, these indices correspond to the usual $\theta, \mathbf{z}$, and $\mathbf{r}$ directions, respectively.) The crystals are assumed to be rate sensitive, with slip rates given by

(1) $\quad \dot{\gamma}=\dot{\gamma}_{0} \frac{\tau}{\tau_{0}}\left|\frac{\tau}{\tau_{0}}\right|$

where $\tau_{0}$ and $\dot{\gamma}_{0}$ are the reference stress and strain rate, and $\tau$ is the resolved shear stress on the slip system considered. Analyses of this type have been employed by Hutchinson ${ }^{2}$, Canova et al. ${ }^{3}$, and Tóth et al. ${ }^{4}$, for instance. The boundary conditions are completed by prescribing zero surface tractions on the plane perpendicular to the 3 axis.

Of course, analytical or semi-analytical solutions can only be obtained in sufficiently simple situations. An example is the case where the set of orientations considered is a trajectory in orientation space; under these conditions, any orientation belonging to the fibre remains on it when deformation proceeds, and a single parameter is sufficient to specify the orientation change. In simple shear, two such cases can immediately be found: orientations with either a $<100>$ or a $<110>$ direction along the 3 axis. Each of these two series of orientations is specified by a single parameter defining the position about the 3 axis, and they are self-symmetric with respect to the symmetry plane of simple shear.

The first fibre was referred to as 'cube-on-face' by Gilormini et al. ${ }^{5}$, and will not be considered any further in the present paper. The second fibre, 'cube-on-edge', is studied in more detail below, with emphasis put on the periodicity of the ODF. It is defined by the angle $\varphi$ between the 1 axis and the $\langle 100\rangle$ direction in the 1-2 plane, see Figure 1a. It contains the $A_{1}{ }^{*}$, $\mathrm{C}$ and $\mathrm{A}_{2}{ }^{*}$ ideal orientations (for $\varphi=35.26^{\circ}, 90^{\circ}$ and $144.74^{\circ}$, respectively, see figure $1 \mathrm{~b}$ ), which are frequently mentioned in the literature on the torsion of fcc polycrystals, since peak values of the ODF are observed experimentally in their vicinities.

\section{ANALYTICAL CALCULATION FOR THE CUBE-ON-EDGE FIBRE}

In the reference $(1,2,3)$ axes, the non-zero components of the prescribed strain rate are $D_{12}=D_{21}=\dot{\gamma} / 2$, and those of the associated stress state are :

$$
\sigma_{11}=-S[\sin 2 \alpha+\sin 2(\alpha-\varphi)] \quad \sigma_{22}=-S[\sin 2 \alpha-\sin 2(\alpha-\varphi)] \quad \sigma_{12}=\sigma_{21}=S \cos 2(\alpha-\varphi)
$$

It is shown below that values of $S$ and $\alpha$ can be found such that the induced slips are responsible for $D$, and that such stress states fulfil the prescribed zero traction condition. (It has already been proved that the solution to this problem is unique, Tóth et al. ${ }^{4}$.)

The resolved shear stresses induced by the above stress state on the 12 slip systems of the FCC structure can now be calculated; the corresponding slip rates are deduced from equation
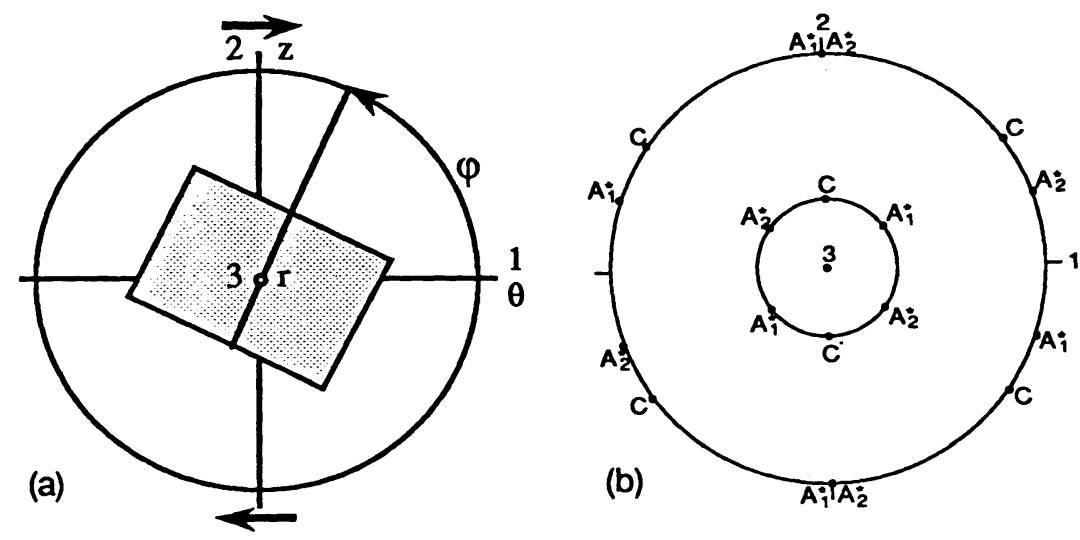

Figure 1 Set of axes (a) and (111) pole figure of the 'cube-on-edge' fibre (b). 
(1) and they are combined to give the resultant strain rate. Comparison with the prescribed strain rate yields the relations that must be satisfied by $\alpha$ and $S$ :

$$
\begin{aligned}
& {[(a+d+2 c+4 b) \sin 2 \varphi-2 \sqrt{2}(a-d) \cos 2 \varphi] \cos 2 \alpha+} \\
& +2 \sqrt{ } 2[(a-d) \sin 2 \varphi-2 \sqrt{ } 2(a+d) \cos 2 \varphi] \sin 2 \alpha=0
\end{aligned}
$$

from which $\alpha$ must be calculated numerically, and

$$
S=\frac{2}{\sqrt{3}} \tau_{0}\left(\frac{3 \sqrt{ } 3}{2 \sqrt{ } 2} \frac{\dot{\gamma}}{\dot{\gamma}_{0}} \sin 2 \varphi\right)^{m}[(a-d) \cos 2 \alpha+2 \sqrt{2}(a+d) \sin 2 \alpha]^{-m}
$$

with $\varphi_{0}=35.26^{\circ}$ and

$$
a=\left|\cos 2\left(\alpha-\varphi_{0}\right)\right|^{1 / m-1} b=\left|\frac{2}{3} \cos 2 \alpha\right|^{1 / m-1} c=\left|\frac{1}{3} \cos 2 \alpha\right|^{1 / m-1} d=\left|\cos 2\left(\alpha+\varphi_{0}\right)\right|^{1 / m-1}
$$

The components of the rate of rotation of the crystallographic axes with respect to the reference directions can then be deduced from the slip rates and from the spin component of the applied deformation. One obtains

$$
\dot{\varphi}=-\frac{\dot{\gamma}}{2}\left[1+\sqrt{3} \sin \left(\varphi_{0}+2 \varphi\right)-\frac{2 \sqrt{2 a} \sin 2 \varphi \cos 2\left(\alpha-\varphi_{0}\right)}{a \cos 2\left(\alpha-\varphi_{0}\right)-d \cos 2\left(\alpha+\varphi_{0}\right)}\right]
$$

The shape of the $\dot{\varphi}(\varphi)$ function for various $m$ values is shown in figure 2a. Note that $\dot{\varphi}$ is negative, i.e. all the orientations on the cube-on-edge fibre move in the direction of the applied shear, and it tends to zero at low $m$ values for the $A_{1}{ }^{*}, C$ and $A_{2}{ }^{*}$ ideal orientations.

Calculation of the ODF intensity variations requires knowledge of the divergence of orientation flow. More precisely, the continuity equation for the ODF intensity $f$ derived by Clément and Coulomb ${ }^{6}$, Clément $^{7}$, and Arminjon ${ }^{8}$ is needed, which is

$$
\dot{\gamma} \frac{\mathrm{d} \ln f}{\mathrm{~d} \gamma}+\dot{\Phi} \cot \Phi+\operatorname{div} \dot{\mathrm{g}}=0
$$

Here a given orientation is followed as its moves through the 3-dimensional Euler space defined by the following angles: a rotation $\varphi$ about the 3-axis (axis 1 rotates to $1^{\prime}$ ) followed by a rotation $\Phi$ about the $1^{\prime}$-axis (axis 3 moves to $3^{\prime}$ ) and a final rotation about the $3^{\prime}$-axis. (The latter rotation is absent in the case of the cube-on-edge fibre, for which $\Phi=45^{\circ}$.) $\mathrm{g}$ denotes the triplet of Euler angles.

There are three terms in the expression for the divergence : the first one represents the variation of $\dot{\varphi}$ when $\varphi$ varies, and it can be obtained from equations (2) and (4). The remain-
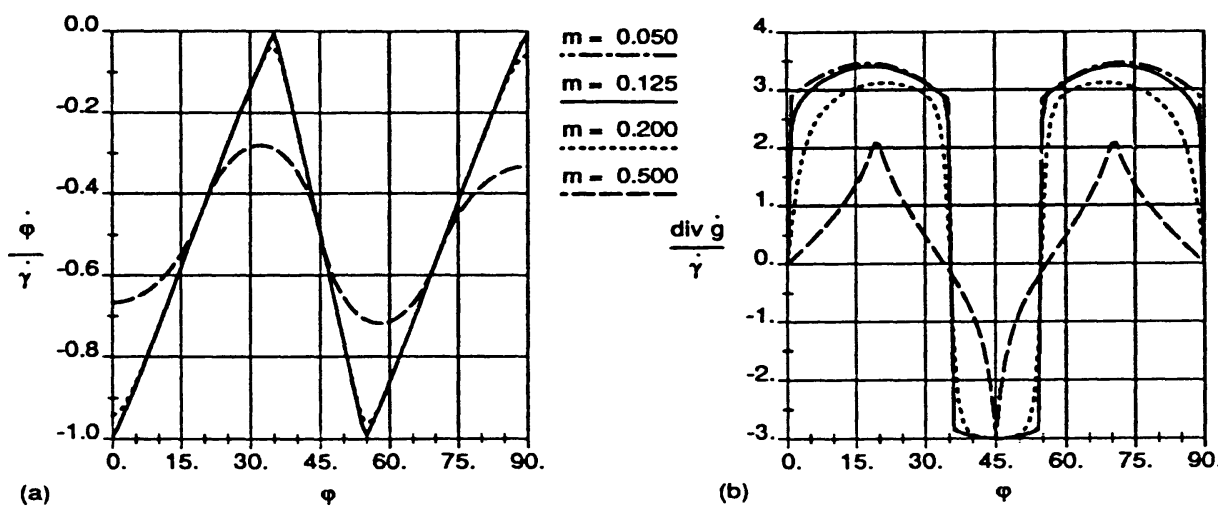

Figure 2 Rotation rate (a) and divergence (b) along the cube-on-edge fibre. 
ning two terms define the variations of the other components of the orientation rate when the two last angles vary. They can be deduced by a first-order analysis around the orientation fibre of interest. The calculations are quite lengthy but not difficult (see Gilormini et al. . $^{5}$ for more detail) ; they lead to the following expression for the divergence along the cube-on-edge fibre:

$$
\begin{aligned}
& \frac{\operatorname{div} \dot{g}}{\dot{\varphi}}=-\sqrt{3} \cos \left(2 \varphi+\varphi_{0}\right)+\frac{2 \sqrt{ } 2(a-d)(b+c) \cos 2 \varphi+[(a+d)(2 b-c)-2 c(2 b+c)] \sin 2 \varphi}{2[a d+(a+d)(b+c)+c(2 b+c)]} \\
& +\frac{2 \sqrt{2 a \cos 2 \varphi \cos 2\left(\alpha-\varphi_{0}\right)}}{a \cos 2\left(\alpha-\varphi_{0}\right)-d \cos 2\left(\alpha+\varphi_{0}\right)}-\frac{256}{9} \frac{\tan 2 \varphi}{\cos 2 \varphi} \frac{a d}{\left[a \cos 2\left(\alpha-\varphi_{0}\right)-d \cos 2\left(\alpha+\varphi_{0}\right)\right]^{2}} \times \\
& \times \frac{2 a d+(a+d)(2 b+c)}{9[(a-d) \tan 2 \varphi-2 \sqrt{2}(a+d)]^{2}-4 a d\left(8-\tan ^{2} 2 \varphi\right)+4(2 b+c)[(a+d+2 b+c) \tan 2 \varphi-2 \sqrt{2}(a-d)] \tan 2 \varphi}
\end{aligned}
$$

The results obtained for various $m$ values are shown in figure $2 b$, from which it can be seen that the divergence is zero for $C$, near $A_{1}$ * and, because of symmetry, near $A_{2}$ *.

Due to the $180^{\circ}$-period of the $\dot{\varphi}(\varphi)$ function, any orientation located on the fibre rotates clockwise $180^{\circ}$ about the 3 -axis for an applied shear equal to

$$
\Gamma=\int_{0}^{-\pi} \frac{\dot{\gamma}}{\dot{\varphi}} \mathrm{d} \varphi
$$

It can be seen from figure 3 that $\Gamma$ increases sharply when $m$ approaches zero; the $\Gamma=2 \pi$ value for $m=1$ was reported previously by Canova et al. ${ }^{3}$ and Tóth et al. ${ }^{4}$. Moreover, since the divergence is also $180^{\circ}$-periodic, equation (5) implies (with $\dot{\phi}=0$ on the cube-on-edge fibre) that the ODF is also $r$-periodic along this fibre for an initially centro-symmetric texture. (The period is $2 \Gamma$ when the initial texture is not centro-symmetric.)

This periodicity could not be determined with the previous combersome computer simulations; it also leads to an interpretation of the oscillatory axial stresses found by Harren et al. ${ }^{9}$ (using a more complicated model including elasticity): the period shown in figure 18 of their paper is about the $\Gamma$ value deduced from the present simple analysis, viz. 6.9 for $m=0.5$, and 12.5 for $m=0.2$. This suggests that the cube-on-edge fibre is responsible for most of the axial stress, since the other orientations are not $\Gamma$-periodic (see Gilormini et al..$^{5}$ ).

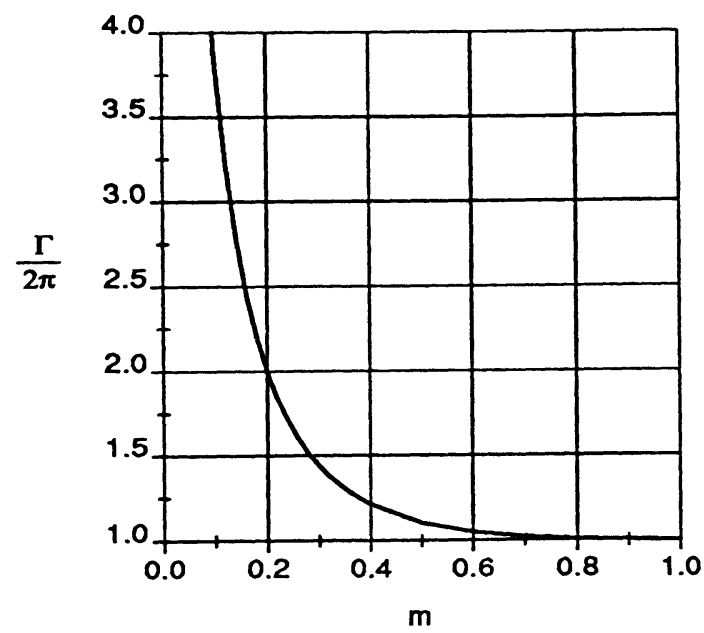

Figure 3 Applied shear value for which a centro-symmetric ODF is restored along the cube-on-edge fibre. 


\section{ODF CALCULATIONS}

Along the cube-on-edge fibre, equation (5) can be integrated easily and gives

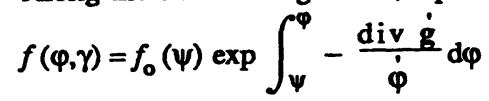

where $f_{0}$ is the initial ODF (for $\gamma=0$ ) and $\psi$ is the initial location of the orientation which travels to the angle $\varphi$ after a shear of $\gamma$. By introducing the following one-variable functions that can be deduced from (4) and (6) and tabulated in the $0-180^{\circ}$ range for any $m$ value,

$$
a(x)=\int_{0}^{x}-\frac{\dot{\gamma}}{\dot{\varphi}} \mathrm{d} \varphi \quad \text { and } \quad f^{*}(x)=\int_{0}^{x}-\frac{\operatorname{div} \dot{g}}{\dot{\varphi}} \mathrm{d} \varphi
$$

equation (9) can be rewritten as

$$
f(\varphi, \gamma)=f_{0}(\psi) \frac{f^{*}(\varphi)}{f^{*}(\psi)}
$$

with

$$
\psi=a^{-1}[\gamma+a(\varphi)]
$$

This allows an immediate and precise calculation of the ODF along the cube-on-edge fibre for any applied shear $\gamma$ without requiring the intermediate $\gamma$ values; thus this method is a good test of the usual incremental computer codes for the simulation of deformation textures. It should be noted that equation (10) shows that an initial ODF defined by $f^{*}$ remains remarkably unchanged along the fibre of interest as deformation proceeds.

Examples of the ODF's predicted along the cube-on-edge fibre by the present method are shown in figure 4 for various $m$ values and for an initially uniform $f=1$ ODF. Note that, due to the periodicity, the ODF for an applied shear of $\Gamma-\gamma$ is obtained from the results at $\gamma$ by symmetry with respect to $\varphi=90^{\circ}$. The peaks near the ideal orientations (defined by dashed lines) appear clearly, and the locations of the tilted peaks considered in Tóth et al. ${ }^{4}$, for instance, can be studied precisely. Whatever the $m$ value, the following developments take place in terms of the period $r$ :

(i) From 0 to $0.32 r$ : three peaks appear, which are shifted first in the sense opposite to the applied shear and then in the shear direction, with intensities such that $A_{1}{ }^{*}<C<A_{2}{ }^{*}$ reaching a maximum when the shift is zero.

(ii) At about $0.32 \Gamma$ : a texture transition occurs; a peak develops rapidly near $\mathrm{C}$, which is shifted opposite to the shear direction.

(iii) From $0.32 \Gamma$ to $0.50 \Gamma$ : smaller peaks appear near $A_{1}{ }^{*}$ and $A_{2}{ }^{*}$, with the intensity of $A_{1}{ }^{*}$ greater than that of $A_{2}{ }^{*}$; the three peaks reach a maximum when the shift, which decreases, is zero.

(iv) At 0.50r: the ODF is symmetric with respect to the maximum at $\varphi=90^{\circ}$, the peak near $A_{1}$ * is shifted in the sense of the applied shear (and that near $\mathrm{A}_{2}^{*}$ in the opposite sense).

Of course, the intensities of the peaks vary with $m$, and they can be extremely high for low $m$ values, but the peaks are very narrow under these conditions; moreover, it should be recalled that the peak intensities grow exponentially in the rate insensitive case.

\section{REFERENCES}

1. K. Wierzbanowski, and A. Clément, Crystal Res.Technol. 19, 201 (1984).

2. J.W. Hutchinson, Proc. R. Soc. Lond. A 348, 101 (1976).

3. G.R. Canova, C. Fressengeas, A. Molinari, and U.F. Kocks, Acta Metall. 36, 1961 (1988)

4. L.S. Tóth,P. Gilormini, and J.J. Jonas, Acta Metall. 36, 3077 (1988).

5. P. Gilormini, L.S. Tóth, and J.J. Jonas, Proc. Roy. Soc. Lond. in press.

6. A. Clément, and P. Coulomb, Scripta Metall. 13, 899 (1979).

7. A. Clément, Mater. Sci. Engng 55, 203 (1982).

8. M. Arminjon, J. Méca. théor. appl. 6, 511 (1987).

9. S. Harren, T.C. Lowe, R.J. Asaro, and A. Needleman, Phil. Trans. Roy. Soc. Lond. A 328, 443 (1989).

10. T.C. Lowe and R.J. Asaro, in Proc. ICOTOM 8, eds. J.S. Kallend and G. Gottstein, The Metallurgical Society, USA, p. 325 (1988). 

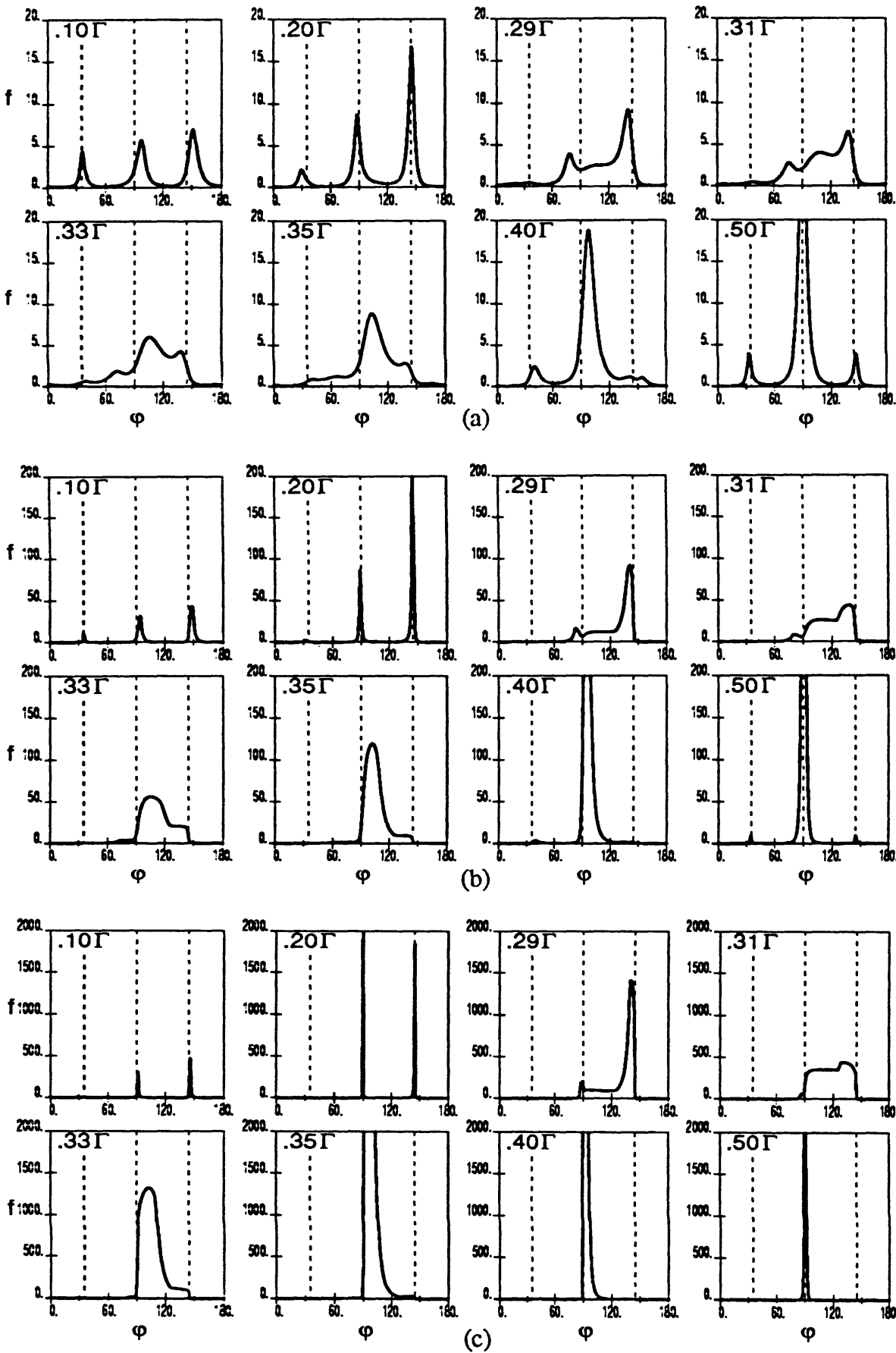

Figure 4 Predicted ODFs for an initially isotropic texture, $m=0.333$ (a), 0.2 (b), 0.125 (c). 